lag $45^{\circ}$ östlich vom Zentralmeridian der Sonne in einer sehr ausgedehnten Fleckengruppe, welche auch an den vorausgehenden Tagen aktiv war.

Diese Zunahmen waren die einzigen, welche die Weißenauer Registrierung im Jahre 1950 verzeichnete. Für eine Störung der Apparate liegen keine Hinweise vor. Aber es ist zunächst nicht verständlich, wieso dieselben in der Registrierung im nur $130 \mathrm{~km}$ entfernten Freiburg nicht auftreten. Sofern es sich um normale Mesonen handelt, dürfte der Effekt in der Kammer maximal 2,5-mal kleiner sein ${ }^{6}$. Er ist aber mindestens 5,5-mal kleiner. Es wäre denkbar, daß es sich um eine mit Hoffmannschen Stößen verknüpfte Strahlung handelt, deren Wirkung in der Kammer mit diesen Stößen eliminiert wurde. Aber zahlenmäßig liegt keine solche Häufung der Stöße vor. Aus dem Verlauf der Effekte wird entgegen den bisherigen Vorstellungen geschlossen und begründet ${ }^{6}$, daß auch diese Zunahmen der Koinzidenzzahl durch magnetische Störfelder verursacht sein können.

\section{Zusatz}

Nach Abschluß obiger Arbeit erhielten wir Kenntnis von einer Arbeit von Sekido und $\mathrm{Yoshida}^{7}$, welche denselben Tagesgang aus Koinzidenzregistrie- rungen statistisch erfaßt hatten, indem sie die Methode der Stichtage auf den Tagesgang anwandten. Sie wählten die Tage mit magnetischen Stürmen als Stichtage und berechneten die mittleren Tagesgänge nach Ortszeit für diese Stichtage, zwei vorausgehende und drei folgende Tage. Die Kurven am 1. und 2. Tag nach den Stürmen gleichen völlig unseren Kurven in Abb. 5 und lassen sich genau so in die beiden Anteile zerlegen. Die Amplitude nimmt von Tag zu Tag etwas ab und springt vom Tag vor dem Sturm zum Stichtag auf doppelte Höhe. Da nur 2 Monate (März und Mai 1948) für die wiedergegebenen mittleren Gänge verwendet wurden und 11 Pfeile den Sturmbeginn an den einzelnen Stichtagen angeben, ist anzunehmen, daß sich das Material überlappt und so die Schwankung vor dem Sturm durch die Statistik zustande kommt, weil die Folge der Stürme manchmal zu dicht war. Die Doppelamplitude macht in diesem gemittelten Material am 1. Tag nach dem Stichtag 0,95\% aus. Am 12. Juni 1948 wurde nach einem Sturmeinsatz in den Morgenstunden eine Gesamtschwankung von $+3 \%$ beobachtet.

7 Yataro Sekido u. Sekiko Yoshida, Report of Ionosphere Research in Japan, Vol. IV, Nr. 1, 1950. Wir danken den Herren für die Übersendung von Separaten.

\title{
Zur Deutung der Tagesgänge der kosmischen Ultrastrahlung bei leichten magnetischen Störungen
}

\author{
Von A. Ehmert \\ Aus der Forschungsstelle für Physik der Stratosphäre in der Max-Planck-Gesellschaft, Weißenau \\ (Z. Naturforschg. 6 a, 622-626 [1951]; eingegangen am 17. August 1951) \\ Erich Regener zum 70. Geburtstag
}

\begin{abstract}
Es wird gezeigt, daß diese Tagesgänge nur auf die zenitnahe Strahlung beschränkt sind, so daß nur eine Beeinflussung energiearmer Primärstrahlen anzunehmen ist. Diese kann durch zur Erde unsymmetrische Magnetfelder bewirkt werden, wie sie von langsamen oder wenig dichten solaren Partikelwolken im Zusammenwirken mit dem Erdfeld entstehen. Eine vorerst hypothetische Möglichkeit einer zusätzlichen Beschleunigung bzw. Verzögerung von Ultrastrahlungsteilchen an der Grenzfläche zwischen dem Erdfeld und der vorbeiflutenden Plasmawolke wird skizziert. - Eine vorläufige Statistik weist auf einen Zusammenhang der Tagesgänge mit Filamenten am Zentralmeridian der Sonne.
\end{abstract}

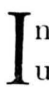
der voranstehenden Mitteilung stellen Ehmert und S ittk us ${ }^{1}$ fest, daß manchmal bei schwachen erdmagnetischen Störungen an mehreren sich folgenden Tagen die Intensität und die Teilchenzahl der

1 A. E h m ert u. A. S it t k u s, Z. Naturforschg. 6a, 618 [1951]; Physik. Verhandlungen Heft 2, 29 [1951].

kosmischen Ultrastrahlung einen auffallenden Tagesgang aufweist, den sie im Mittel sogar in zwei getrennte Effekte zerlegen können: nämlich in einen recht genau einem Sinusgang folgenden Anteil mit der Periode von $24 \mathrm{Stdn}$. und dem Maximum kurz nach Mittag (Ortszeit) und in eine baiartige Ein- 
buchtung der Intensität, welche am frühen Nachmittag (Ortszeit) scharf einsetzt, gegen $17 \mathrm{Uhr}$ ihre maximale Elongation erreicht und bis Mitternacht ausklingt.

Die einfache Amplitude der Sinusschwingung beträgt für das Mittel von 10 Tagen mit den ausgeprägtesten Effekten bei der Zählrohrkoinzidenzanlage $A_{\mathrm{K}}=0,6 \%$, bei der Ionisationskammer $A_{\mathrm{I}}=0,4 \%$. Die Baitiefe macht bei den Koinzidenzen $B_{\mathrm{K}}=$ $0,74 \%$, bei der Ionisationskammer $B_{\mathrm{I}}=0,32 \%$ aus.

Es wird zunächst gezeigt, daß man diesen Zahlen entnehmen muß, daß an der Sinusschwingung nur Teilchen aus einem Winkelbereich von etwa $30^{\circ} \mathrm{um}$ den Zenit beteiligt sind, während die Teilchen, welche bei der baiartigen Störung ausfallen, aus Winkelbereichen $<10^{\circ}$ um den Zenit stammen sollten.

In Abb. 1 zeigt die Kurve F für unsere Koinzidenzanlagen in Abhängigkeit vom Zenitwinkel $\alpha$ bei Mittelung über alle Azimute das Verhältnis der wirksamen Registrierfläche zur Registrierfläche für senkrechten Einfall. Setzt man entsprechend den Meßergebnissen $^{2}$ die Intensitätsverteilung proportional $\cos ^{2} \alpha$ an und berücksichtigt, daß (in räumlichen Polarkoordinaten) das Raumwinkelelement mit dem Zenitwinkel $\alpha-\frac{\mathrm{d} \alpha}{2}$ bis $\alpha+\frac{\mathrm{d} \alpha}{2}$ die Größe $2 \pi \cdot \sin \alpha \cdot \mathrm{d} \alpha$ hat, so ergibt die in Abb. 1 gezeichnete Kurve $V_{\mathrm{K}}(\alpha)=$ $F(\alpha) \cdot \sin \alpha \cdot \cos ^{2} \alpha$ die Verteilung der registrierten Strahlen über den Zenitwinkel $\alpha$. Bei der Ionisationskammer ist das wirksame Volumen von der Strahlenrichtung unabhängig. An die Stelle der Funktion $F(\alpha)$ tritt hier eine Konstante $=1$. Die Verteilung der in der Ionisationskammer registrierten Strahlen über die Zenitrichtung ergibt deshalb die in Abb. 1 gestrichelt eingetragene Kurve $V_{\mathrm{I}}(\alpha)=\sin \alpha \cdot \cos ^{2} \alpha$. Nehmen nun die im Bereich $0 \leq \alpha \leq \alpha_{0}$ einfallender Strahlen um $a \% \mathrm{ab}$, so wird man in beiden Fällen die relativen Effekte registrieren:

$$
\begin{gathered}
e_{\mathrm{K}}=\frac{a \int_{0}^{a_{0}} F(\alpha) \sin \alpha \cos ^{2} \alpha \mathrm{d} \alpha}{\int_{0}^{\pi / 2} F(\alpha) \sin \alpha \cos ^{2} \alpha \mathrm{d} \alpha} \\
\text { und } e_{\mathrm{I}}=\frac{a \int_{0}^{a_{0}} \sin \alpha \cos ^{2} \alpha \mathrm{d} \alpha}{\int_{0}^{\pi / 2} \sin \alpha \cos ^{2} \alpha \mathrm{d} \alpha},
\end{gathered}
$$

und man erhält das Größenverhältnis der relativen Effekte

2 z. B. A. E h m e r t, Physik. Z. 35, 20 [1934].

$$
\frac{e_{\mathrm{K}}}{e_{\mathrm{I}}}=\frac{\int_{0}^{a_{0}} V_{\mathrm{K}}(\alpha) \mathrm{d} \alpha}{\int_{0}^{a_{0}} V_{\mathrm{I}}(\alpha) \mathrm{d} \alpha} \quad 2,47 \mathrm{mit} \frac{\int_{0}^{\pi / 2} V_{\mathrm{I}}(\alpha) \mathrm{d} \alpha}{\int_{0}^{\pi / 2} V_{\mathrm{K}}(\alpha) \mathrm{d} a}=2,47
$$

Die Zahl 2,47 ist eine Apparatkonstante dieser speziellen Zählrohranordnung. Die Größe $a$ tritt in dem Verhältnis $e_{\mathrm{K}} / e_{\mathrm{I}}$ nicht mehr auf.

Bei sehr kleinem $\alpha_{0}$ sind $V_{\mathrm{K}}$ und $V_{\mathrm{I}}$ identisch, so daß dann $e_{\mathrm{K}} / e_{\mathrm{I}}$ den Maximalwert 2,47 erreicht. Mit wachsendem $\alpha_{0}, \mathrm{~d}$. h. wachsendem, beeinflußtem

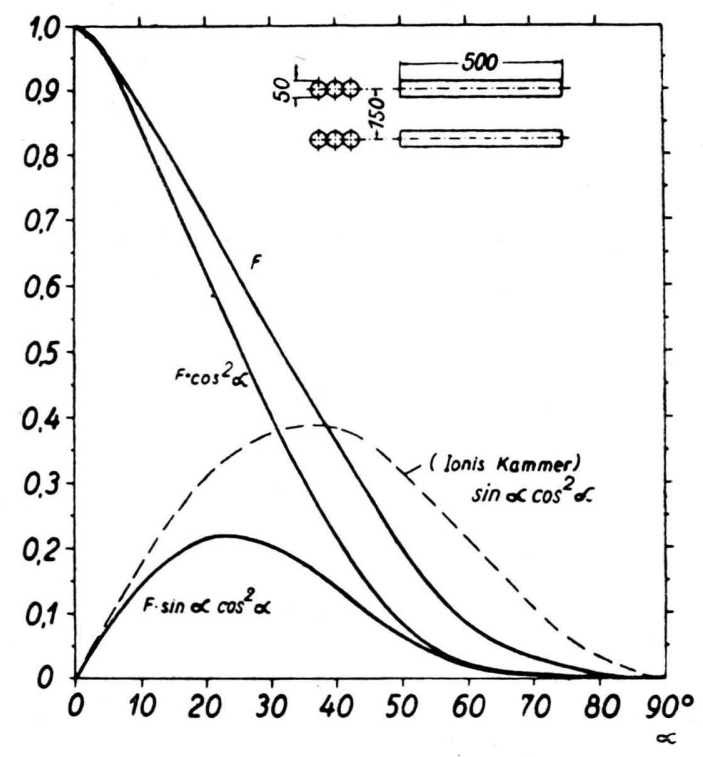

Abb. 1. Die Verteilung der Empfindlichkeit $F$ und der registrierten Intensität über die Zenitwinkel für die Ionisationskammer und für die Koinzidenzanlage, die im Bild skizziert ist.

Winkelbereich, nimmt dieses Verhältnis ab bis zum Wert 1 bei $\alpha_{0}=90^{\circ}$. Das Verhältnis $e_{\mathrm{K}} / e_{\mathrm{I}}=1,5$, wie es für die Amplituden der Sinuswelle gefunden wurde, ist schon bei $\alpha_{0}=36^{\circ}$ erreicht. In Wirklichkeit mag kein allzu scharfes Abschneiden eintreten und so der Winkelbereich der für die Sinuswelle verantwortlichen Strahlung etwas größer sein.

Das für die baiartige Störung bei besonders ausgeprägtem Tagesgang gefundene Verhältnis $B_{\mathrm{K}} / B_{\mathrm{I}}=$ 2,32 entspricht nur einem $\alpha_{0}$ von $5^{\circ}$. Bei der $\mathrm{Zu}-$ sammenfassung von 11 Tagen mit weniger ausgeprägtem Tagesgang ergab sich sogar sowohl für $A$ als auch für $E$ nahezu das theoretische Verhältnis für Strahlung aus engsten Winkelbereichen. Der Schluß auf eine enge Bündelung ist zwingend. Wenn die 
Ionisationskammer noch einen merklichen Anteil einer nicht mit $\cos ^{2} \alpha$ zenitabhängigen Strahlung (z. B. den Resteffekt) mitmißt, so wird die Apparatkonstante nicht 2,47, sondern etwas größer, und wir haben dann zu kleine Divergenzwinkel berechnet.

Der Vergleich mit einer Koinzidenzanlage anderer Apertur könnte diesen Zweifel völlig klären. Aber es ist wenig Einfluß auf das Resultat zu erwarten, daß der Tagesgang auf Strahlung sehr enger Winkelbereiche zurückgeht und daß eine Zunahme der Sinuswellenamplitude mit einer Verbreiterung dieses Winkelbereiches verknüpft ist. Damit kann das verschiedene Amplitudenverhältnis der beiden Schwankungen in den Registrierungen sinnvoll gedeutet werden:

Es wird nur das energiearme Ende des Energiespektrums der primären Ultrastrahlung wesentlich beeinflußt. Hier reicht in unseren Breiten die Energie schon knapp aus, um Mesonen zu erzeugen, welche den Erdboden erreichen. Diese erliegen außerdem einer Schwächung durch den Zerfall auf wenige Prozente der ursprünglichen Zahl. Mit wachsendem Zenitwinkel nimmt die Wahrscheinlichkeit für zum Boden durchdringende Mesonen bei derselben Primärenergie sehr rasch weiter $a b$, so daß sich die Beeinflussung der zugehörigen schräg einfallenden Primären am Boden nicht mehr meßbar auswirken kann.

Die Panzerung der Ionisationskammer absorbiert ebenfalls Mesonen, welche bei der Koinzidenzanlage noch zur Messung kommen. Es sind nur wenige Prozent, weil diese Mesonen geringer Energie in der Troposphäre durch Zerfall dezimiert werden. Für die Effekte der normalen Strahlung wird deshalb das Amplitudenverhältnis $e_{\mathrm{K}} / e_{\mathrm{I}}$ wenig verändert.

Arders wäre dies für eine eventuelle zusätzliche Strahlung, welche durch einen anderen Absorptionsund Transmissionsmechanismus durch die Atmosphäre gelangt und dabei starker örtlicher Absorption unterliegt. Hier könnte das Effektverhältnis $e_{\mathrm{K}} / e_{\mathrm{I}}$ den Wert 2,5 übersteigen. Dies ist ein weiterer Hinweis darauf, daß die Tagesgänge mit dem normalen Verhältnis durch normale Mesonen aus Primären geringer Energie zustande kommen.

Es ist zu bedenken, daß bei einem Effekt von $a \%$ der gesamten registrierten Strahlung bei Beschränkung des Effektes auf einen Zenitwinkelbereich von

3 Man vergleiche die zusammenfassende Darstellung von J. B a r t e l s , Z. Geophysik 14, 297 [1938].

$4 \mathrm{~S}$. C h a p m a n, Nature [London] 140, 423 [1937].

5 Vgl. die Monographie: S. C h a p m a n u. J. B a r tels, Geomagnetism Bd. I, Clarendon Press, Oxford 1940. $20^{\circ}$ innerhalb dieses Bereichs dann der Effekt etwa $3 a \%$ ausmachen muß. Weitere Messungen mit enger Öffnung der Koinzidenzanlage sollen diesen Punkt prüfen.

Der weltweite Charakter der Tagesgänge und ihre Verbindung mit leichten erdmagnetischen Störungen legen die Zurückführung derselben auf weitreichende magnetische Felder um die Erde nahe. Der Versuch, sie mit den magnetischen Feldern der ebenfalls ortszeitbedingten ionosphärischen Ströme ${ }^{3}$ zu erklären, scheitert, weil ja diese Ströme in Erdnähe bleiben müssen und deshalb zwischen dem Störfeld und der Amplitude des Tagesganges eine bleibende Korrelation bestehen müßte. Ebenso könnte die tägliche Erwärmung der Stratosphäre nur einen gegenphasigen Effekt hervorrufen.

Weltweit sind aber auch die bei erdmagnetischen Stürmen auftretenden Abnahmen der Intensität der kosmischen Ultrastrahlung. $\mathrm{C} \mathrm{h}$ a p m a ${ }^{4}$ führte diesen Zusammenhang auf die Ablenkung von Ultrastrahlungsteilchen im Magnetfeld eines Ringstromes um die Erde zurück, der bei starken magnetischen Stürmen entsteht ${ }^{5}$. Das Feld dieses Ringstromes ist nach Großstörungen tagelang zu beobachten und ist als „Nachstörung“ (zur Hauptphase des magnetischen Sturmes) besonders deutlich an äquatorialen Stationen während der Nacht zu erkennen, weil nachts die Ionosphärenströme ausfallen und am Äquator die zusätzlichen Störströme der Polarlichtzonen fehlen. Nach der Großstörung am 26. Juli 1946 yerlief die in mehreren Tagen langsam abklingende erdmagnetische „Nachstörung“ des Ringstromes, wie wir sie den Nachtwerten von Huancayo (Peru, $4^{\circ} \mathrm{S}$ ) entnehmen ${ }^{6}$, praktisch ebenso wie die Störung der kosmischen Ultrastrahlung in Cheltenham nach Forbush ${ }^{7}$ und in England nach Elliot und Dolbear ${ }^{8}$. Wir finden 1\% Abnahme der Ultrastrahlung pro $16,4 \gamma$ Verminderung der Horizontalintensität in Huancayo. Die Hauptphase des magnetischen Sturmes hatte in diesem Fall keine stärkere Abnahme der Ultrastrahlung verursacht. Bei anderen magnetischen Stürmen, wie z. B. am 1. 3. 1942, zeigte die Ultrastrahlung während der Hauptphase eine zusätzliche Abnahme, welche etwa die gleiche Größe hatte wie der maximale Ringstromeffekt. Dieser Einfluß der Hauptphase scheint um so größer zu sein, je rascher dieselbe auf die

6 Herrn Prof. B a rtels danke ich für die freundliche Vermittlung und Darstellung der Werte.

7 S. E. F o r b u s h, Physic. Rev. 70, 771 [1946].

8 D. W. N. D olb e a r u. H. Elli ot, Nature [London] 159, 58 [1947]. 
chromosphärische Eruption folgt, d. h. je schneller die Partikelströme sind, welche die magnetische Störung auslösen. Es muß jedoch noch mehr Material abgewartet werden. Mehrere Autoren verglichen die Ultrastrahlung mit Feldregistrierungen aus hohen Breiten, wobei die eben genannten einfachen $\mathrm{Zu}-$ sammenhänge durch Felder von Strömen in der Polarlichtzone verdeckt wurden.

Ursache der großen magnetischen Stürme sind Partikelwolken, welche von der Sonne bei starken chromosphärischen Eruptionen fortgestoßen werden können. Sie haben eine Querausdehnung von der Ordnung $10^{10} \mathrm{~cm}$ und eine Geschwindigkeit von 1000 bis $2000 \mathrm{~km} / \mathrm{sec}$. Sie treffen die Erdbahn nach 20 bis 40 Stunden Laufzeit. Sie sind sicher neutral, jedoch ionisiert, also von hoher Leitfähigkeit und können nach neueren Vorstellungen auch ein inneres Magnetfeld besitzen. Die Teilchendichte wird in Erdnähe auf bis zu $10^{3}$ Teilchen $/ \mathrm{cm}^{3}$ geschätzt.

Ein so rasch bewegtes Plasma kann in das magnetische Erdfeld nicht eindringen, es sei denn in Richtung der Kraftlinien, weil sonst Wirbelströme entstehen und diese die Bewegung abbremsen. Deshalb muß nach $\mathrm{Ch}$ a pman und Ferrar ${ }^{9}$ um die Erde im Plasmastrom ein Hohlraum entstehen, dessen Wände auf beiden Seiten der Erde außerdem infolge der Polarisation des Plasmas durch das Feld eine Oberflächenladung aufweisen sollten. Im Ausgleich dieser Ladung hinter der Erde vermuten $\mathrm{Ch}$ a p m an und Ferraro ${ }^{9}$ das Zustandekommen des Ringstroms, dessen Natur und Aufbau keineswegs geklärt sind. Er scheint so hohe Stabilität anzunehmen, daß er sich noch lange hält, wenn die Erde sich nicht mehr im Plasmastrahl befindet. Er verursacht ein zusätzliches Magnetfeld, das innerhalb des Ringstromes das Erdfeld schwächt, außerhalb aber eine Verstärkung bedeutet, sobald die Plasmawolke abgezogen ist oder nur noch wesentlich kleinere Geschwindigkeit aufweist als am Anfang. Im Inneren der Wolke wird ja das äußere Magnetfeld durch Wirbelströme abgeschirmt.

Ein solcher beständiger Ringstrom muß weitgehend symmetrisch zur Erde sein. Offensichtlich muß zu seinem Zustandekommen die Partikelwolke ausreichende Dichte und Geschwindigkeit haben. Im anderen Fall kommt es nur zu einem unsymmetrischen Hohlraum im Plasma, der jedoch weit größer sein kann als der Ringstromdurchmesser. Deshalb sind die

9 S. Chapman u. V. C. A. F e rraro, Terrestr. Magnetism and Atmospheric Electricity 36, 77 [1931].

10 C. S tö r m e r, Astrophysica Norvegica II, Nr. 4 [1937]. erdmagnetischen Wirkungen klein. Ihr genauer Mechanismus kann heute nicht angegeben werden, doch ist die Annahme von Unsymmetrien zur Erde plausibel. Diese können durch einen Gradienten der Teilchendichte quer zum Teilchenstrahl noch verstärkt werden. Solche Unsymmetrien sind annähernd raumfest zur Linie von der Sonne zur Erde, womit sich die Ortszeitbindung ihres Einflusses auf die kosmische Ultrastrahlung erklären läßt.

Wir vermuten, daß die Tagesgänge auf diese unsymmetrischen Felder zurückgehen. Dafür spricht insbesondere die meist die Tagesgänge einleitende schwache Partikelstörung, welche sich erdmagnetisch nur durch ihre Wirkung in der Polarlichtzone ausdrückt. Dorthin können die Partikel entlang den Kraftlinien des Erdfeldes kommen.

Im Gegensatz zum Ringstrom komprimiert die Teilchenwolke durch ihre Wirbelströme das Erdfeld im Hohlraum der Wolke um die Erde; am stärksten auf der Mittagsseite der Erde, wo die Wolkenelemente direkt auf das Erdfeld stoßen. Von dort her kommen aber die um Mitternacht zur Zeit des Minimums bei uns einfallenden energiearmen Ultrastrahlen $(H \varrho \approx$ $\left.1,2 \cdot 10^{7} \mathrm{Gau} ß \cdot \mathrm{cm}\right)$, denn sie werden im Erdfeld nach $\mathrm{S}$ tör m e r ${ }^{10}$. um etwa $180^{\circ}$ umgelenkt.

Die Einsattelung der Tagesgänge am Nachmittag deutet in diesem Zusammenhang auf eine erhöhte abschirmende Wirkung auf die Ultrastrahlung aus der Richtung, nach welcher sich die Erde bei ihrer Bahn um die Sonne bewegt. Die Geschwindigkeit der Erde ist mindestens um eine Größenordnung kleiner als die Wolkengeschwindigkeit. Aber die Bewegung des Feldes senkrecht zu den Kraftlinien und gegen die Polarisationsladung der Oberfläche des Hohlraums im Plasma kann einen zusätzlichen Effekt hervorrufen.

Das betrachtete Störfeld um die Erde ist von derselben Art wie das Störfeld während der Hauptphase starker Stürme, bevor es zur Ausbildung eines Ringstromes kommt. Die durch dieses Feld bewirkte Abnahme der Ultrastrahlung ist in manchen Fällen doppelt so groß wịe der anschließende Ringstromeffekt ${ }^{11}$ und scheint nur in den Nachmittags- und Abendstunden aufzutreten. Ihre relative Größe ist bei Koinzidenzanordnungen größer als bei Ionisationskammern, während der Ringstromeffekt in beiden Anordnungen fast die gleiche prozentuale Abnahme bewirkt. Darin zeigt sich die Unsymmetrie dieses Feldes ${ }^{12}$. Es hört auf, wenn die Erde die Plasmawolke

11 S. z. B. A. Ehmert, Z. Naturforschg. 3a, 264 [1948] Abb. 2.

12 Eine quantitative Diskussion wird a. a. Ort gegeben. 
verläßt. Bei starken Stürmen wirkt sich die Hauptphase auch am Äquator auf die Ultrastrahlung aus ${ }^{13}$.

Bei schwachen Partikelstörungen kann die Erde tagelang von Plasmawolken umgeben sein. Wenn es jedoch zur Ausbildung eines Ringstromes kommt, werden die energiearmen Primärteilchen im Feld desselben ohnehin abgelenkt und die Tagesgänge hören auf, wie es der Beobachtung entspricht.

Wie weit elektrische Felder im Plasmahohlraum mitspielen, ist schwer zu übersehen. Nach $\mathrm{Ch}$ a p $\mathrm{m}$ a $\mathrm{n}$ und Ferrar o soll von der Sonne aus gesehen die rechte Wand des Plasmahohlraums eine negative, die linke Wand (gegen welche sich die Erde bewegt) eine positive Ladung haben, also in groben Zügen ein elektrisches Feld entgegen der Erdbahn entstehen. Dieses wird eben durch die Bewegung der nachdringenden Wolke aufrechterhalten. Das Erdfeld verhindert den direkten Ausgleich, bei dem sich die Ionen senkrecht zu den magnetischen Kraftlinien bewegen müßten. Die Teilchen können nicht anlaufen. Aber die schon magnetisch steif von außen kommenden Ultrastrahlungsteilchen können das Feld durchlaufen und dabei etwas beschleunigt oder verzögert werden. Das daraus resultierende Maximum wäre aber in den Nachtstunden zu erwarten, wo wir ein Minimum beobachten.

Derartige Einflüsse könnten zu einem Tagesgang führen, welcher im Maximum über den ungestörten Normalwert hinausgeht, während die magnetische Ablenkung allein dies nicht bewerkstelligen kann. Anzeichen für solche Effekte sind in geringer Zahl vorhanden.

Wenn die Plasmawolke selbst ein Magnetfeld mit sich führt, wäre sogar an die Möglichkeit zu denken, daß auch an der Wand des Hohlraums um die Erde Ultrastrahlung beschleunigt bzw. verzögert wird, ähnlich wie Ki epenheuer ${ }^{14}$ die Erzeugung der solaren Ultrastrahlung am Rand der Plasmawolken vermutet, wenn diese die Korona verlassen haben und in so dünn mit Ionen besetzte Gebiete kommen, daß die Energie der das Magnetfeld 'in der Wolke nach außen abschirmenden Wirbelströme auf nur verhältnismäßig wenige Ionen übertragen wird, welche dann eben diese Wirbelströme darstellen. Mit anderen

13 In Tokio schneidet das Erdfeld das Ultrastrahlungsspektrum bei $H \varrho=4 \cdot 10^{7} \mathrm{Gau} \beta \cdot \mathrm{cm}$ ab. Vgl. den Zusatz bei $\mathrm{Ehmert}$ und $\mathrm{S}$ it tku s${ }^{1}$.
Worten: Entlang der mit einem Magnetfeld behafteten Wolke soll ein elektrisches Induktionsfeld in Richtung der Bewegung entstehen, das von den Ionen in seiner ganzen Ausdehnung durchlaufen werden soll, wobei diese Ionen in ihrer Gesamtheit die Abschirmung des Feldes auf ihrer Bahn übernehmen müssen.

Ähnliche Verhältnisse liegen am Rand der Plasmawolke gegen das Erdfeld vor. Wenn auch hier die Energie nicht zur Erzeugung von neuen Ultrastrahlen ausreichen wird, welche das Erdfeld zu durchdringen vermögen, so kann doch der Vorgang für die Entstehung des Ringstromes von Bedeutung sein und eine zusätzliche Beschleunigung von Ultrastrahlungsteilchen bewirken, welche je nach der Feldorientierung in der Wolke etwa um Mittag oder Mitternacht die Erde erreichen können, nachdem sie im Erdfeld nochmals umgelenkt werden.

Wir sind uns des durchaus hypothetischen Charakters der gegebenen Deutungen bewußt. Aber wir sahen hier die erste Möglichkeit eines Zusammenhanges zwischen Messungen auf der Erde und den Vorgängen am Rand des Magnetfeldes der Erde und möchten sie zur Diskussion stellen. Um so mehr als damit die unerwarteten Einzelheiten dieser Tagesgänge wenigstens qualitativ mit bekannten Erscheinungen in plausible Beziehung gebracht werden können. Mögen diese experimentellen Tatsachen ein Anreiz zur Weiterführung der einschlägigen Theorien sein.

Es sei noch bemerkt, daß eine vorläufige, noch durch weiteres Material zu ergänzende Statistik des Auftretens der Tagesgänge auf einen Zusammenhang mit Filamenten weist. Dies sind hohe flächenhafte Protuberanzen, welche bei Aufsicht auf die Sonnenscheibe in $\mathrm{H} \alpha$ durch ihre Absorption fadenähnlich aussehen ${ }^{15}$. Sie können nach Eruptionen in ihrer Nähe zum Aufsteigen kommen und teilweise die Sonne verlassen. Fast bei allen sehr auffallenden Tagesgängen hatten sehr auffallende Filamente den Zentralmeridian passiert. So z. B. am 16. bis 18. Febr. 1950 , am 24. bis 26. Febr. 1950, am 15. bis 18. März 1950, am 16. und am 27. und 28. Sept. 1950, am 8. bis 11. Jan. 1951, am 17. bis 19. Febr. 1951, am 24. und 25. Febr. 1951 und am 7. bis 9. März 1951.

14 K. O. Ki e p e n h e u e r, Physic. Rev. 78, 809 [1950].

15 Vgl. M. W a ld mei er, Ergebnisse und Probleme der Sonnenforschung. Leipzig 1941. 